\title{
COMPORTAMENTO DO CRESCIMENTO DE Chlorella sorokiniana EM ESCALA LABORATORIAL E AMPLIADA
}

\author{
V. O. ARAÚJO' ${ }^{1}$, G. C. MONTANHIM ${ }^{2}$, A. T. LOMBARDI ${ }^{3}$ \\ ${ }^{1}$ Universidade Federal de São Carlos, Laboratório de Biotecnologia de Algas \\ ${ }^{2}$ Universidade Federal de São Carlos, PPGERN \\ ${ }^{3}$ Universidade Federal de São Carlos, Departamento de Botânica \\ E-mail para contato: vini.quim@gmail.com
}

\begin{abstract}
RESUMO - Microalgas são organismos fotossintetizantes, cuja biomassa possui alto valor agregado, já que é rica em biomoléculas atrativas a vários ramos da indústria. Além disso, têm sido organismos alvo de empresas no processo de mitigação de carbono. O cultivo em larga escala ainda apresenta muitos desafios para os pesquisadores, como alto custo do processo como um todo, coleta da biomassa e fatores abióticos limitantes. Assim, a modelagem matemática pode proporcionar respostas para variáveis utilizadas durante um cultivo. O objetivo do presente trabalho foi representar parâmetros de biomassa por um modelo com comportamento exponencial, ajustando os parâmetros a fim de descrever o crescimento da microalga em relação ao tempo. Foram realizados cultivos da microalga Chlorella sorokiniana em escala laboratorial e ampliada. Com o auxílio do software Igor aplicou-se o ajuste de Boltzman utilizando-se os dados de clorofila $a\left(\mathrm{mg} \mathrm{L}^{-1}\right)$ e densidade óptica, mensurados diariamente. $\mathrm{O}$ modelo empregado descreveu o crescimento da microalga nas fases exponencial e estacionária de crescimento, mostrando-se adequado e evidenciado tais estágios de crescimento.
\end{abstract}

\section{INTRODUÇÃO}

As microalgas planctônicas apresentam atributos biotecnológicos tal qual rápida velocidade de crescimento e versatilidade metabólica, tornando-as organismos promissores para produção comercial de compostos com alto valor agregado (BOROWITZKA e MOHEIMANI, 2013).

Microalgas são uma fonte atrativa de biomassa para a produção de energia e biomoléculas, já que não competem com a agricultura na produção de alimentos e possuem maior rendimento energético por área do que as plantações terrestres tradicionais. No entanto, o sistema de cultura, o enriquecimento com nutrientes, necessários na formulação do meio nutritivo e o sistema de coleta de biomassa, podem tornar-se fatores impeditivos à viabilidade econômica da produção de biomassa algal (CLARENS et al., 2010; LUNDQUIST et al., 2010).

Considerando o comportamento do cultivo e a produtividade, a modelagem matemática desempenha a condição de oferecer respostas para parâmetros utilizados. O desenvolvimento 
de equacionamentos que depende de variáveis do processo aumenta a capacidade de serviço enquanto especifica a condição de melhor trabalho para as variáveis do processo.

Para a produção industrial destinada ao ganho de biomassa, saber o ponto ótimo de operação é economicamente mais favorável devido à maior produção em menor tempo sem perda de produto. Segundo Galvão et al. (2011) o aumento populacional em culturas algais com volume limitado possui comportamento de uma curva sigmoidal. O modelo utilizado analisa todos os pontos do período do desenvolvimento microbiano e fornece a taxa de crescimento para o período até atingir a fase estacionária. A vantagem de empregar a equação de Boltzmann (1872) é que para crescimentos microbianos onde a fase exponencial não é muito bem definida o ajuste fornece os parâmetros de crescimento como a velocidade de propagação e ponto máximo da curva.

\section{OBJETIVOS}

Representar parâmetros de biomassa, a saber, concentração de clorofila $a$ e densidade óptica por um modelo com comportamento exponencial, no qual os parâmetros serão ajustados com a finalidade de descrever o crescimento da microalga ao longo do tempo.

\section{MATERIAIS E MÉTODOS}

Em escala laboratorial, sob condições controladas de intensidade luminosa (130 $\mu$ mol s $\mathrm{s}^{-}$ $\left.{ }^{1} \mathrm{~m}^{-2}\right)$, fotoperíodo claro-escuro $(12: 12 \mathrm{~h})$ e temperatura $\left(24{ }^{\circ} \mathrm{C} \pm 1{ }^{\circ} \mathrm{C}\right)$ foram utilizados frascos de policarbonato com capacidade de $1000 \mathrm{~mL}$ e $200 \mathrm{~mL}$ para o cultivo de $800 \mathrm{~mL}$ e $80 \mathrm{~mL}$ de Chlorella sorokiniana, respectivamente. Utilizou-se o meio de cultura BG 11 (RIPPKA et al., 1979) modificado.

Em escala ampliada, foram utilizados fotobiorreatores de plástico e de vidro, respectivamente com 100 e 1000 litros de cultivo. Os ensaios foram realizados no pátio do Laboratório de Biotecnologia de Algas, da UFSCar. A agitação foi desempenhada por bombas submersas $\left(2000 \mathrm{~L} \mathrm{hora}^{-1}\right)$, sendo três bombas para $1000 \mathrm{~L}$ e uma para $100 \mathrm{~L}$. Houve borbulhamento de $\mathrm{CO}_{2}$ e de ar atmosférico.

As culturas foram monitoradas diariamente para os parâmetros densidade óptica (espectrofotômetro digital Macherey-Nagel, Nanocolor UVIVIS, Alemanha), densidade celular (contagem de células sob microscópio óptico em hemocitômetro Fuchs-Rosenthal e contador automático (Muse ${ }^{\mathrm{TM}}$ Cell Analyzer, Alemanha)), concentração de clorofila $a$ por determinação da fluorescência in vivo (Turner Designs, Model Trilogy - USA), e eficiência fotossintética por meio da de fluorescência da clorofila utilizando-se um aparelho Phyto-PAM (Walz, Alemanha) de acordo com metodologia descrita em Lombardi e Maldonado (2011).

Para se evidenciar o fato de curvas de crescimento de microalgas apresentarem característica sigmoidal, com auxílio do software Igor aplicou-se o ajuste de Boltzman (1872), equação 1 , utilizando os dados de clorofila $a\left(\mathrm{mg} \mathrm{L}^{-1}\right)$ e densidade óptica, mensurados diariamente. 


$$
y(t)=\frac{B}{1+e^{-(t-r) \cdot \mu}}+A
$$

em que

$y(t)=$ Concentração clorofila a $\left[\mathrm{mg} \mathrm{L}^{-1}\right]$ ou absorbância.

$\mathrm{B}=$ ponto máximo da curva, com mesma unidade de $\mathrm{y}(\mathrm{t})$.

$\mathrm{A}=$ valor inicial, com mesma unidade de $\mathrm{y}(\mathrm{t})$.

$\mathrm{t}=$ variável tempo [tempo]

$\tau=$ parâmetro que determina o ponto de inflexão da curva [tempo].

$\mu=$ taxa de crescimento [tempo ${ }^{-1}$.

\section{RESULTADOS E DISCUSSÃO}

A curva de crescimento da microalga $C$. sorokiniana possui comportamento sigmoidal, assim como o modelo previsto por Verhulst (1838) para o crescimento populacional biológico auto limitante. Desta forma, para funções sigmoides habitualmente utilizadas a equação (1) de Boltzman (1879) expressou melhor ajuste com os dados de parâmetros de biomassa da espécie microalgal.

\section{A equação}

$$
y(t)=\frac{B}{1+e^{-(t-\tau) \cdot \mu}}+A
$$

No qual y(t) representa a concentração de clorofila a $\left[\mathrm{mg} \mathrm{L}^{-1}\right.$ ou Abs], A corresponde a concentração inicial [mg clorofila a $\mathrm{L}^{-1}$ ou Abs] e B [mg clorofila a $\mathrm{L}^{-1}$ ou $\left.\mathrm{Abs}\right], \tau[\mathrm{h}]$ e $\mu\left[\mathrm{h}^{-1}\right]$ são parâmetros da equação. Para o presente trabalho foi assumido que a vaiável $\mu$ é constante e fisicamente equivalente à taxa de crescimento obtida por mensuração da clorofila $a$ ou de densidade óptica, descrevendo, portanto, a taxa de crescimento da microalga em relação ao tempo. O parâmetro $\tau$ reflete o ponto de inflexão da curva e caracteriza o instante do tempo em que a concavidade da curva muda para baixo.

As curvas para o modelo e os dados experimentais estão representados nas figuras 1 e 2 . As informações para os valores dos parâmetros do ajuste são encontradas nas tabelas 1 e 2 .

O modelo utilizado descreve o crescimento da microalga na fase exponencial e na fase estacionária para as variáveis respostas que podem expressar a concentração celular. No entanto, a equação não consegue esboçar o período de decaimento celular. A fim de assegurar que as condições fisiológicas saudáveis estavam sendo mantidas, para todo o intervalo de obtenção de dados a condição fisiológica da microalga foi avaliada e verificou-se que as células mantiveram-se saudáveis, de acordo com a metodologia descrita em Lombardi e Maldonado (2011), como podemos verificar na figura 3.

Para o ajuste empregado é importante analisar os parâmetros $\mathrm{B}$ e $\mu$ em conjunto. A constante $\mathrm{B}$ é o ponto máximo da curva e a variável $\mu$ é a velocidade de crescimento. Como mostrado nas tabelas 1 e 2 , a taxa de crescimento é independente do ponto máximo da curva, ou melhor, quanto mais alta a velocidade de crescimento, mais rapidamente a curva alcançará o ponto ótimo para, posteriormente, chegar à fase estacionária. 
Muitos são os trabalhos que envolvem modelos matemáticos para expressar dados referentes à biomassa microalgal.

Figura 1 - Modelo e dados experimentais para a concentração de clorofila $a$ de $C$. sorokiniana para cultivos em escala laboratorial e ampliada em relação ao tempo.

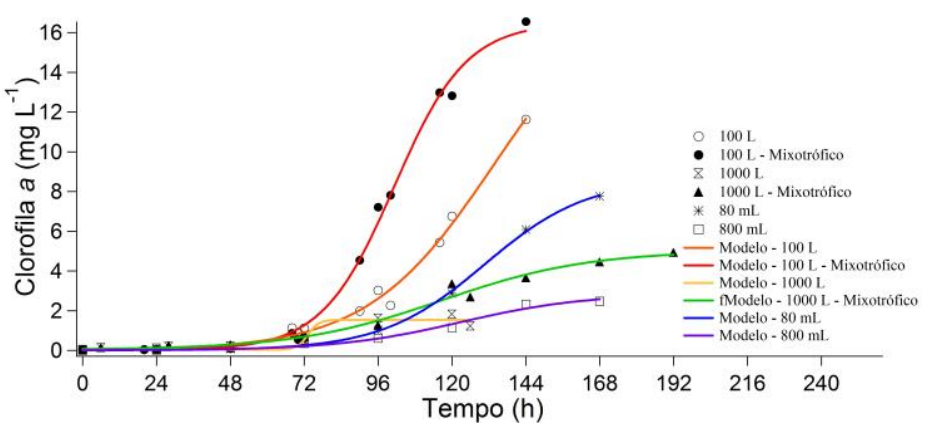

Sukenik et al. (1991) elaboraram um modelo para avaliar o efeito da profundidade do reservatório e da produção de clorofila $a$ na taxa de produção de biomassa de Isochrysis galbana em várias estações.

Figura 2 -Modelo e dados experimentais para absorbância $(684 \mathrm{~nm})$ de C. sorokiniana para cultivos em escala laboratorial e ampliada em relação ao tempo.

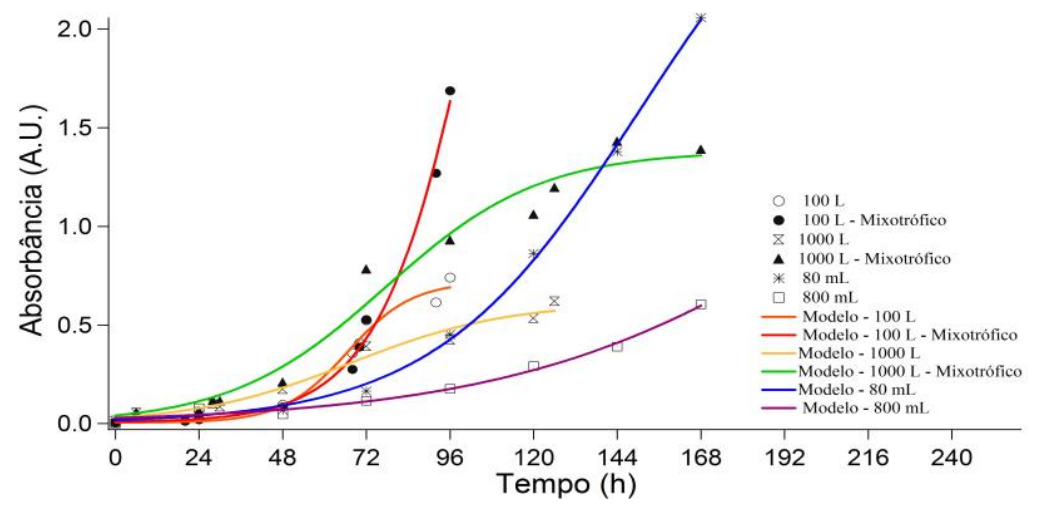

Figura 3 - Rendimento fotossintético máximo de C.sorokiniana para cultivos em escala laboratorial e ampliada em relação ao tempo.

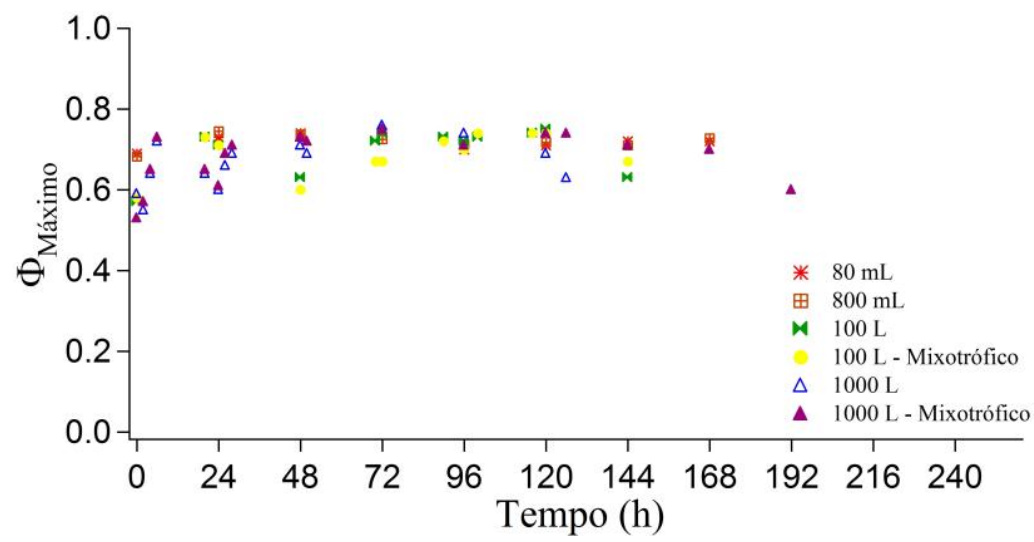


Grima et al. (1994), propuseram um modelo matemático para o crescimento contínuo de uma cultura microalgal limitada por luz, utilizando o coeficiente de absorção da biomassa. O modelo reproduziu os estados estacionários atingidos e o comportamento dinâmico do sistema.

Syed Shabudeen et al. (2013) verificaram o crescimento de Botryococcus braunii, Chlorella species e Chlorella vulgaris sob as suas condições ótimas de crescimento e a utilização de dados cinéticos para a concepção e operação do sistema de cultivo em grande escala, utilizando a variável densidade óptica.

Yu et al. (2011) realizaram um cultivo mixotrófico com Anabaena sp, por meio da adição de glicose, o que aumentou a densidade celular e a taxa específica de crescimento. Houve variação na composição de pigmentos celulares, entre eles a clorofila $a$. Um modelo de Monod modificado foi desenvolvido para descrever o crescimento celular, incorporando a taxa específica de crescimento e a intensidade de luz média no reator.

Tabela 1 - Valores dos parâmetros da equação (1) para concentração de clorofila A em cada tratamento.

\begin{tabular}{|c|c|c|c|c|c|c|}
\hline Parâmetros & $180 \mathrm{~L}$ - Mixo & $180 \mathrm{~L}$ - Photo & $1000 \mathrm{~L}$ - Photo & $1000 \mathrm{~L}$ - Mixo & $80 \mathrm{~mL}$ & $800 \mathrm{~mL}$ \\
\hline \hline A [mg/L] & 0.0178 & 0.0180 & 0.0373 & 0.033 & 0.006 & 0.005 \\
\hline B [mg/L] & 16.487 & 18.782 & 1.505 & 4.992 & 8.643 & 2.844 \\
\hline$\tau[\mathrm{h}]$ & 101.25 & 133.84 & 73.20 & 116.79 & 1330.55 & 122.90 \\
\hline$\mu\left[\mathrm{h}^{-1}\right]$ & 0.085346 & 0.048386 & 0.558004 & 0.042012 & 0.059726 & 0.049488 \\
\hline$\mu\left[\mathrm{dia}^{-1}\right]$ & 2.048305 & 1.161271 & 13.39210 & 1.008276 & 1.433435 & 1.187707 \\
\hline
\end{tabular}

Tabela 2 - Valores dos parâmetros da equação (1) para absorbância em cada tratamento.

\begin{tabular}{|c||c|c|c|c|c|c|}
\hline Parâmetros & $180 \mathrm{~L}$ - Mixo & $180 \mathrm{~L}$ - Photo & $1000 \mathrm{~L}$ - Photo & $1000 \mathrm{~L}-$ Mixo & $80 \mathrm{~mL}$ & $800 \mathrm{~mL}$ \\
\hline \hline A [mg/L] & 0.0070 & 0.0063 & 0.0016 & 0.0013 & 0.0017 & 0.0030 \\
\hline B [mg/L] & 5.39 & 0.71 & 0.61 & 1.38 & 3.13 & 2.30 \\
\hline$\tau[\mathrm{h}]$ & 108.41 & 66.57 & 67.49 & 77.53 & 149.63 & 220.28 \\
\hline$\mu\left[\mathrm{h}^{-1}\right]$ & 0.07 & 0.11 & 0.04 & 0.04 & 0.03 & 0.02 \\
\hline$\mu\left[\mathrm{dia}^{-1}\right]$ & 1.62 & 2.58 & 1.06 & 1.08 & 0.83 & 0.48 \\
\hline
\end{tabular}

\section{CONCLUSÕES}

O modelo empregado mostrou-se adequado para as variáveis que consideram biomassa algal, neste caso, concentração de clorofila $a$ e absorbância.

Muitos são os casos de espécies microalgais em que alguma das fases de crescimento não ficam evidentes em representações gráficas convencionais. Assim, o modelo empregado neste trabalho facilitou o entendimento do comportamento de Chlorella sorokiniana em determinadas condições, podendo também ser aplicado em outras espécies de microalga. 


\section{AGRADECIMENTOS}

Agradeço à professora $\mathrm{Dr}^{\mathrm{a}}$ Ana Teresa Lombardi, ao apoio financeiro concedido, aos colegas do Laboratório de Biotecnologia de Algas e ao professor Dr. César Rogério de Oliveira, do Departamento de Matemática da UFSCar.

\section{REFERÊNCIAS}

BOLTZMANN, L.E. Weitere Studien über das Wärmegleichgewicht unter Gasmolekülen, Wiener Berichte 66,1, 306-402, 1872.

BOROWITZKA, M.A.; MOHEIMANI, N.R. 2013. Mitig Adapt Strateg Glob Change, 18: 47-72.

CLARENS, A.F.; RESURRECCION, E.P.; WHITE, M.A.; COLOSI, L.M. Environmental life cycle comparison of algae to other bioenergy feedstocks. Environ. Sci \& Techn. 44, 1813 -1819. 2010.

GALVÃO, R.M.; SANTANA, T.S.; FONTES, C.H.O.; SALES, E.A. Estudo da taxa de crescimento de microorganismos e proposta de modelo para produção de biomassa de Haematococcus pluvialis. Anais do III International workshop advances in cleaner production, 2011.

GRIMA, E.M.; CAMACHO, F.G.; PÉREZ, J.A.S.; SEVILLA, J.M.F.; FERNÁNDEZ, F.G.A.; GÓMEZ, A.C. A mathematical model of microalgal growth in light-limited chemostat culture. 1994. J. Chem. Tech. Biotechnol., 61:167-173.

LOMBARDI, A. T.; MALDONADO, M.T. The effects of copper on the photosynthetic response of Phaeocystis cordata. Photos. Res., 108: 77-87, 2011.

LUNDQUIST, T.J.; WOERTZ, I.C.; QUINN, N.W.T.; BENEMANN, J.R. A Realistic Technology and Engineering Assessment of Algae Biofuel Production. Energy Biosciences Institute, 1-178, 2010.

RIPPKA, R.; DERUELlES, J.; WATERBURY, J.; HERDMAN, M.; STANIER, R. Generic assigments, strain histories and properties of pures cultures of cyanobacteria. J. Gen. Microbiol, 111, 1-61, 1979.

SUKENIK, A.; LEVY, R.S.; LEVY, Y.; FALKOWSKI, P.G.; DUBINSKY, Z. Optimizing algal biomass production in an outdoor pond: a simulation model. 1991. J. Appl. Phyc. 3: 191-201.

SYED SHABUDEEN, P.S.; SOUNDRARAJAN, M.; INDUMATHI, P. Algae biomass growth kinetic study in waste water medium using spectroscopi analysis. J. Environ. Res. Develop. 7, 4A:14961500, 2013.

VERHULST, P.F. Notice sur la loi que la population poursuit dans son accroissement. Corresp. Math. Phys. 10, 113-121 (1838). books.google.com Disponível em: http://webpages.fc.ul.pt/ mcgomes/aulas/dinpop/Mod13/Verhulst.pdf Acesso em 18 de março de 2017.

YU, G.; SHI, D.; CAI, Z.; CONG, W.; OUYANG, F. Growth and physiological features of cyanobacterium Anabaena sp. strain PCC 7120 in a glucose-mixotrophic culture. 2011, Chin. J. Chem. Eng., 19:108-115. 